\title{
Methylcrotonyl-CoA and geranyl-CoA carboxylases are involved in leucine/isovalerate utilization (Liu) and acyclic terpene utilization (Atu), and are encoded by liuB/liuD and atuC/atuF, in Pseudomonas aeruginosa
}

\begin{abstract}
Correspondence
Dieter Jendrossek

dieter.jendrossek@imb.uni-

stuttgart.de
\end{abstract}

Received 10 June 2005

Revised 29 July 2005

Accepted 5 August 2005

\author{
Birgit Höschle, ${ }^{1}$ Volker Gnau ${ }^{2}$ and Dieter Jendrossek ${ }^{1}$ \\ ${ }^{1}$ Institut für Mikrobiologie, Universität Stuttgart, Allmandring 31, 70550 Stuttgart, Germany \\ ${ }^{2}$ Universität Tübingen, Tübingen, Germany
}

\begin{abstract}
Pseudomonas aeruginosa is able to grow on acyclic monoterpenes (citronellol, citronellate, geraniol and geranylate), and on other methyl-branched compounds such as leucine or isovalerate. The catabolic pathway of citronellol (Atu, acyclic terpene utilization) enters that of leucine/ isovalerate (Liu, leucine and isovalerate utilization) at the level of methylcrotonyl-CoA. Key enzymes of the combined pathways are geranyl-CoA carboxylase (GCase) and methylcrotonyl-CoA carboxylase (MCase). In this study, isovalerate-grown cells specifically expressed MCase (apparent molecular mass of the biotin-containing subunit, $74 \mathrm{kDa}$ ) only, and the GCase biotin-containing subunit $(71 \mathrm{kDa})$ was not detected. Citronellol- or citronellate-grown cells produced both carboxylases. Biotin-dependent proteins were purified from crude extracts by avidin-affinity chromatography, and assigned to the corresponding coding genes by trypsin fingerprint analysis. The two subunits of MCase corresponded to liuB/liuD (PA2014/PA2012) of the $P$. aeruginosa genome database, and atuC/atuF (PA2888/PA2891) encoded GCase subunits. This finding is contrary to that reported by others. The identified genes are part of two separate gene clusters [liuRABCDE (PA2011-PA2016) and atuABCDEFGH (PA2886-PA2893)] that are thought to encode most of the genes of the Atu and Liu pathways.
\end{abstract}

\section{INTRODUCTION}

Citronellol (3,7-dimethyloct-6-en-1-ol) and geraniol (3,7dimethylocta-2,6-dien-1-ol) are model compounds of acyclic monoterpenes, which occur naturally in plants. Citronellol and geraniol are of industrial interest due to their commercial use in the food (flavour) and perfume (odour) industries. Moreover, citronellol is one of very few naturally occurring repellents of insects (mosquitoes) (Hierro et al., 2004; Pawar et al., 1991; Rice \& Coats, 1994). Geraniol is structurally related to citronellol, and differs only by the presence of an additional double bond. Recently, it was found that geraniol and related compounds have significant effects on eukaryotic and human cells, and are able to induce apoptosis in vitro in pancreatic cancer cells (Burke et al., 1997, 2002; Carnesecchi et al., 2002a, b, 2004; Duncan et al., 2004; Izumi et al., 1999). The intensity and variety of biological activities of monoterpenes and related compounds have not been fully investigated. A lack of information on

Abbreviations: Atu, acyclic terpene utilization; GCase, geranyl-CoA carboxylase; Liu, leucine and isovalerate utilization; MCase, methylcrotonyl-CoA carboxylase. the biochemical routes of monoterpenes in organisms might be one reason for the shortage of knowledge. Citronellol is the only acyclic monoterpene for which some information exists on its biochemistry in micro-organisms. This knowledge is based mainly on investigations performed by Seubert and coworkers with Pseudomonas citronellolis more than 40 years ago (Seubert, 1960; Seubert et al., 1963; Seubert \& Remberger, 1963; Seubert \& Fass, 1964a, b), and these were continued by Fall and coworkers in the late 1970s (Cantwell et al., 1978; Fall \& Hector, 1977; Fall et al., 1979; Hector \& Fall, 1976a, b). The first steps of the catabolic pathway of citronellol and geraniol are the oxidation of the alcohols to citronellate or geranylate, and subsequent activation to the corresponding CoA esters citronellyl-CoA and geranyl-CoA. Due to the structural similarity of citronellol and geraniol, it is assumed that oxidation of citronellol and geraniol is catalysed by the same enzymes (Fig. 1). However, recent data suggest that oxidation of geranial and/or geraniol is dependent on molybdenum, and that the reaction is different from molybdenum-independent oxidation of citronellol (Höschle \& Jendrossek, 2005). Citronellyl-CoA can be converted to geranyl-CoA by a dehydrogenase step, and all 


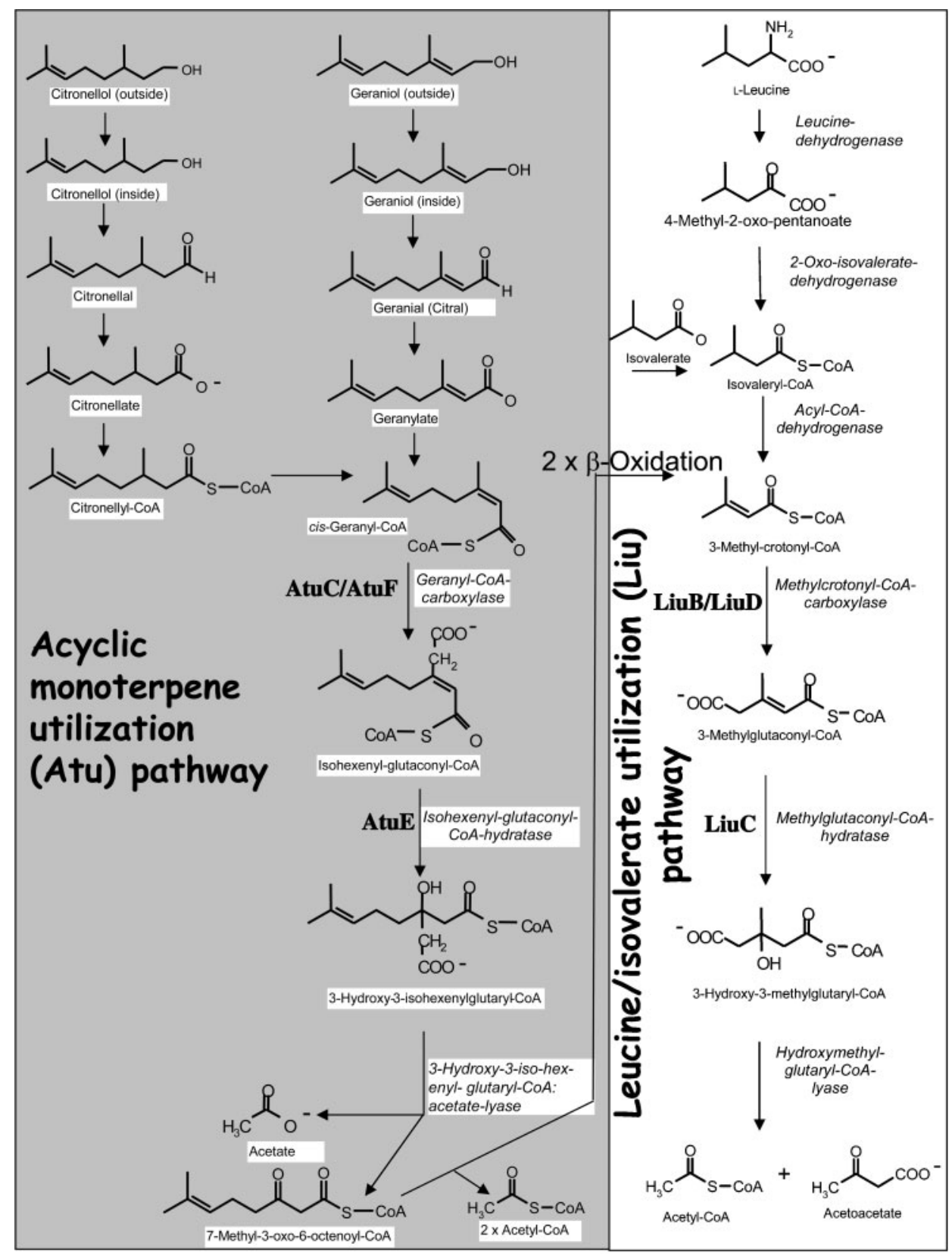

Fig. 1. Putative Atu and Liu pathways in $P$. citronellolis, according to Seubert \& Fass (1964a). Genes of $P$. aeruginosa PAO1 identified in this study (atuC/atuF and liuB/liuD), and putative isohexenyl-glutaconyl-CoA hydratase (atuE) and methylglutaconyl-CoA hydratase (liuC) genes, are indicated.

subsequent reactions are the same for citronellol and geraniol utilization. Geranyl-CoA is difficult to metabolize because of the presence of $\beta$-methyl groups that inhibit $\beta$ 3 -oxidation. The key enzyme of the citronellol/geraniol degradation pathway is geranyl-CoA carboxylase (GCase) (Seubert et al., 1963). GCase converts the branched-chain $\beta$-methyl group of geranyl-CoA to an acetate group that can be cleaved off more easily than a methyl group. For details of the catabolic pathway of citronellol/geraniol see Fig. 1 .
The metabolite 7-methyl-3-oxo-6-octenoyl-CoA can be degraded by two rounds of subsequent $\beta$-oxidation reactions, without hindrance by $\beta$-methyl groups. The resulting metabolite (3-methylcrotonyl-CoA) is degraded via the leucine/isovalerate utilization (Liu) pathway (Fig. 1), which involves another carboxylase (methylcrotonyl-CoA carboxylase, MCase) that is unable to utilize geranyl-CoA as a substrate, but is able to utilize carboxylate methylcrotonylCoA (Fall \& Hector, 1977; Fall, 1981; Hector \& Fall, 1976a, 
b). The two branched-chain-specific carboxylases GCase and MCase have been purified and partially characterized in P. citronellolis (Fall \& Hector, 1977).

Pseudomonas aeruginosa and Pseudomonas mendocina are two additional bacteria with the capacity to utilize citronellol and other linear terpenes (Cantwell et al., 1978). Recently, utilization of citronellol by $P$. aeruginosa was investigated by Diaz-Perez and coworkers (Diaz-Perez et al., 2004). Those authors cloned a gene cluster consisting of six genes (PA2011-PA2016) that are necessary for growth on linear terpenes in $P$. aeruginosa. The cluster contained two genes (gnyA/gnyB, PA2012/PA2014) for putative carboxylase subunits, and other genes that possibly encode proteins of the acyclic (mono-) terpene utilization (Atu) pathway. In our present study, we initiated investigation of the Atu pathway by a biochemical approach, and we came to different conclusions than those of Diaz-Perez and coworkers.

\section{METHODS}

Bacterial strains, plasmids and culture conditions. The bacterial strains and plasmids used in this study are shown in Table 1. Cultures of $P$. aeruginosa PAO1 were routinely grown in LuriaBertani (LB) medium, or in mineral salt medium (Schlegel et al., $1961)$ containing different carbon sources $[0 \cdot 1 \%(\mathrm{v} / \mathrm{v})$ sodium citronellate, $0 \cdot 1 \%(\mathrm{v} / \mathrm{v})$ sodium geranylate or $0 \cdot 1 \%(\mathrm{v} / \mathrm{v})$ isovaleric acid], at $30{ }^{\circ} \mathrm{C}$. Water-insoluble carbon sources (citronellol and geraniol) were added in the space between the Petri dish and the agar $(20 \mu \mathrm{l}$ per plate), and the plates were sealed with Parafilm. Growth on solid media with liquid carbon sources was performed in separate incubators to avoid cross-contamination by vapours. Liquid cultures contained $0.5 \%$ glucose or $0.1 \%$ glucose, and either $0 \cdot 2 \%$ sodium citronellate or $0 \cdot 2 \%$ isovalerate. The Escherichia coli strain was grown in LB medium at $37^{\circ} \mathrm{C}$. Solid media contained $1.5 \%(\mathrm{w} / \mathrm{v})$ agar.

For conjugation experiments, P. aeruginosa $\mathrm{PAO} 1 \mathrm{Sm}^{\mathrm{r}}$ and E. coli S171 , containing the respective plasmids, were grown in LB medium. Equal volumes of the donor and the recipient cultures were mixed, spotted onto an LB agar plate, and incubated for $6 \mathrm{~h}$ at $37^{\circ} \mathrm{C}$. The cells were resuspended in $0.5 \mathrm{ml}$ saline, plated on selective agar containing $500 \mu \mathrm{g}$ streptomycin $\mathrm{ml}^{-1}$ and $50 \mu \mathrm{g}$ gentamicin $\mathrm{ml}^{-1}$, and incubated overnight at $37^{\circ} \mathrm{C}$.

Insertion mutagenesis. Disruption of atuF (PA2891) in P. aeruginosa was carried out using pKnockout-G for rapid gene inactivation (Windgassen et al., 2000). A 3 '- and 5'-truncated fragment of atuF was obtained by PCR-mediated amplification of atuF from $P$. aeruginosa genomic DNA using the synthetic oligonucleotides PA2891-EcoRIFwd (5'-GGAATTCCGCGCCGAGCTGATCCTGGAGAGG-3') and PA2891-EcoRI-Rev (5'-GGAATTCCCTGGGGTCTTCGGCGAAGCGTTC-3') as primers. The EcoRI-digested purified 778 bp PCR fragment of $a t u F$ was cloned in the EcoRI site of pKnockout-G, and transformed in E. coli S17-1. The plasmid pKnockout-G::atuF was transferred to $P$. aeruginosa $\mathrm{PAO} 1 \mathrm{Sm}^{\mathrm{r}}$ via conjugation, and transconjugants were selected on LB agar containing $500 \mu \mathrm{g}$ streptomycin $\mathrm{ml}^{-1}$ and $50 \mu \mathrm{g}$ gentamicin $\mathrm{ml}^{-1}$. The disruption of $a t u F$ was verified by (i) PCR using one $a t u F$-specific primer and one pKnockoutspecific primer, and (ii) two Southern blot hybridization experiments of chromosomal mutant DNA with a DIG-labelled probe specific for the gentamicin-resistance gene, and a DIG-labelled probe specific for atuF.

\begin{abstract}
Isolation of biotin-containing proteins, Western blot analysis and trypsin fingerprint analysis. Late-exponential cells were collected by centrifugation at $4{ }^{\circ} \mathrm{C}$, and washed with mineral salt medium that did not contain a carbon source. The pellet was resuspended in $25 \mathrm{mM}$ Tris/ $\mathrm{HCl}$ buffer, $\mathrm{pH} 7 \cdot 5$, at $1 \mathrm{ml}$ (g cells) ${ }^{-1}$. The suspension was passed two times through a French press cell at 800 p.s.i. $(5 \cdot 5 \mathrm{MPa})$. Cell debris and membrane fragments were removed by centrifugation at $80000 \mathrm{~g}$ in a Beckman rotor Ti50 for $1 \mathrm{~h}$ at $4{ }^{\circ} \mathrm{C}$. The supernatant was used for further analysis. The isolation of biotin-containing proteins was done with immobilized monomeric avidin. A small column was packed with $250 \mu \mathrm{l}$ immobilized monomeric avidin, and equilibrated with PBS containing $0 \cdot 1 \mathrm{M}$ phosphate buffer, $0 \cdot 15 \mathrm{M} \mathrm{NaCl}, \mathrm{pH} 7 \cdot 0$. A $250 \mu \mathrm{l}$ volume of the crude extract was applied to the column, which was then washed six times with 1 vol. PBS. Biotin-containing proteins were eluted from the column with elution buffer ( $2 \mathrm{mM}$ D-biotin in PBS). The column was regenerated by washing two times with $0 \cdot 1 \mathrm{M}$ glycine $\mathrm{HCl}, \mathrm{pH} 2 \cdot 8$.
\end{abstract}

Samples were separated by $10 \%$ SDS-PAGE, and either stained with Coomassie blue, or transferred to PVDF membranes for Western blot analysis. Protein bands of interest were cut out, and subjected to trypsin digestion and subsequent LC-MS/MS analysis. Western blotting was done by standard procedures. The blotted biotin-containing proteins were tagged with Streptavidin-AP conjugate (Roche), and the colour development was done with nitroblue tetrazolium chloride (NBT) and 5-bromo-4-chloro-3-indolyl-phosphate $p$-toluidine salt (BCIP). Prestained dual-colour SDS-PAGE standard proteins (Bio-Rad) were used as marker proteins.

Nanoflow liquid chromatography tandem MS (nano-LC-MS/ MS). Reverse-phase nano-LC-MS/MS was performed using an Ultimate nanoflow LC system (LC Packings). The LC system was coupled to a QSTAR Pulsar hybrid qTOF mass spectrometer (ABMDS Sciex), which used a modified nanoelectrospray ion source (Proxeon Biosystems) interface. Briefly, the tryptic peptide mixtures

Table 1. Bacterial strains and plasmids

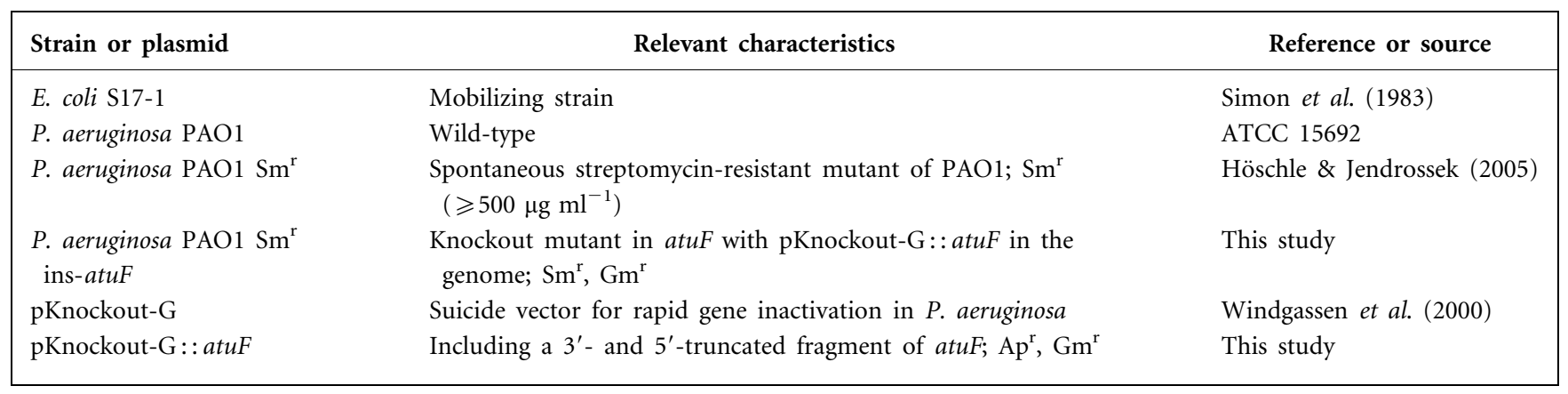


were autosampled at a flow rate of $50 \mu \mathrm{min}^{-1}$ in $0 \cdot 1 \%$ aqueous trifluoroacetic acid, and desalted on a PepMap C18 trapping cartridge (LC Packings). The trapped peptides were eluted and separated on the analytical column (PepMap C18, $75 \mu \mathrm{m}$ i.d. $\times 15 \mathrm{~cm}$; LC Packings) using a linear gradient of $7-50 \%$ acetonitrile in $0.5 \%$ formic acid for $30 \mathrm{~min}$ at a flow rate of $200 \mathrm{nl} \mathrm{min}{ }^{-1}$, and ionized by an applied voltage of $2 \cdot 2 \mathrm{kV}$ to the emitter.

The mass spectrometer was operated in data-dependent acquisition mode to automatically switch between MS and MS/MS. Survey MS spectra were acquired for $1 \mathrm{~s}$, and the three most intense ions (doubly, triply or quadruply charged) were isolated, and sequentially fragmented for $1.5 \mathrm{~s}$ by low-energy collision-induced dissociation. All MS and MS/MS spectra were acquired with the Q2-pulsing function switched on, and optimized for enhanced transmission of ions in the MS $(\mathrm{m} / \mathrm{z}$ $400-1000)$ and MS/MS ( $m / z 75-1300)$ mass ranges.

\section{RESULTS}

Biotin-containing proteins of $P$. aeruginosa PAO1 were purified from (i) glucose-, (ii) citronellate- and (iii) isovalerate-grown cells, separated by reducing SDS-PAGE (Fig. 2A), and screened for biotin by Western blot analysis (Fig. 2B), as described in Methods. Only one biotincontaining polypeptide (apparent molecular mass $63 \mathrm{kDa}$ ) was found in glucose-grown cells. This protein was constitutively expressed, and apparently is not involved in carboxylation of methyl-branched compounds (see below). One additional biotin-containing polypeptide appeared at about $74 \mathrm{kDa}$ in isovalerate-grown cells. This protein presumably corresponds to the biotin-containing subunit of MCase, because the catabolic pathway of leucine/isovalerate involves only one carboxylase step (MCase). Specific induction of MCase by leucine and isovalerate has been shown for P. citronellolis (Fall \& Hector, 1977; Hector \& Fall, 1976a, b). In citronellate-grown cells of $P$. aeruginosa, a third biotindependent protein at about $71 \mathrm{kDa}$ appeared in addition to the 63 and $74 \mathrm{kDa}$ proteins (Fig. 2B). Since GCase is necessary only in citronellol/citronellate-grown cells, but not in isovalerate-grown cells, the $71 \mathrm{kDa}$ protein is thought to correspond to GCase.

The purified biotin-containing proteins of citronellategrown cells (Fig. 2A) were cut out from the Coomassiestained SDS-polyacrylamide gel, and subjected to trypsin fingerprint analysis and ESI-MS-MS (double or multiple mass spectra)-based amino acid sequencing. At least 13 peptides of each isolated biotin-containing protein were analysed, and compared with the $P$. aeruginosa database. The $63 \mathrm{kDa}$ biotin band (band no. 3, Fig. 2A) perfectly matched to the putative product of ORF PA5435 (putative acetyl-CoA carboxylase/transcarboxylase subunit), and the $74 \mathrm{kDa}$ (no. 1) and $71 \mathrm{kDa}$ (no. 2) biotin-containing proteins were unequivocally identified as the gene products of liuD (PA2012; $74 \mathrm{kDa}$ ) and $a t u F(P A 2891 ; 71 \mathrm{kDa})$, respectively (Figs 2 and 3 ). The three non-biotin-containing polypeptides (band nos 4/5 and 6, Fig. 2A) corresponded to liuB (PA2014), atuC (PA2888) and the product of PA5436,

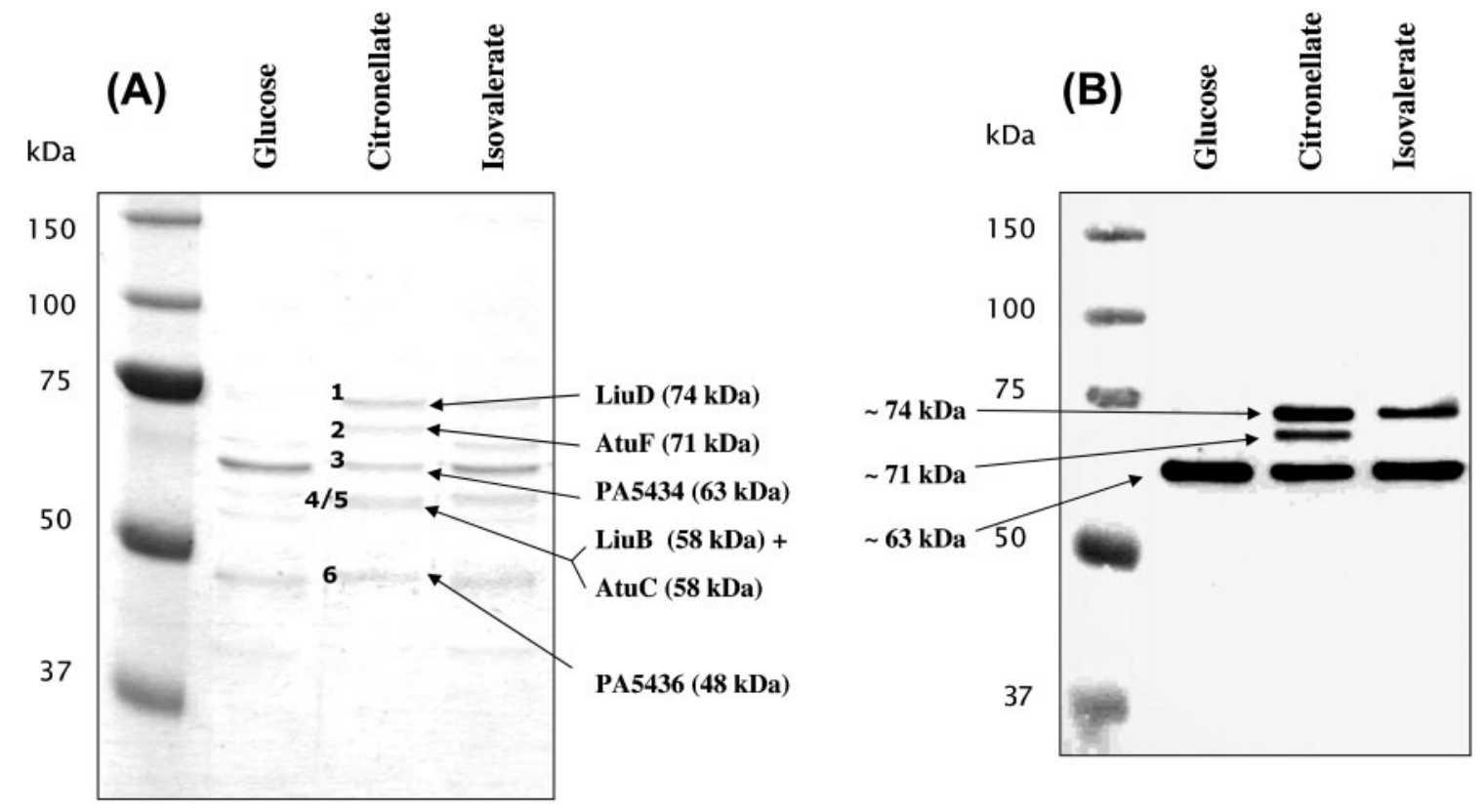

Fig. 2. SDS-PAGE and Western blot analysis of $P$. aeruginosa PAO1 for biotin-containing proteins. After avidin purification, soluble cell extracts were separated on $10 \%$ SDS-polyacrylamide gels and stained with Coomassie blue (A), or transferred to PVDF membranes and screened for biotin-containing proteins (B), as described in Methods. To obtain sufficient resolution in the range above $50 \mathrm{kDa}$, electrophoresis was carried out for about $90 \mathrm{~min}(20-25 \mathrm{~mA})$ so that proteins of less than $\sim 25 \mathrm{kDa}$ had run out of the gel. Gene products identified by trypsin fingerprint LC-MS analysis of isolated protein bands are indicated. The numbering of the protein bands in $(A)$ is described in the text. 
(A) P. aeruginosa atu cluster

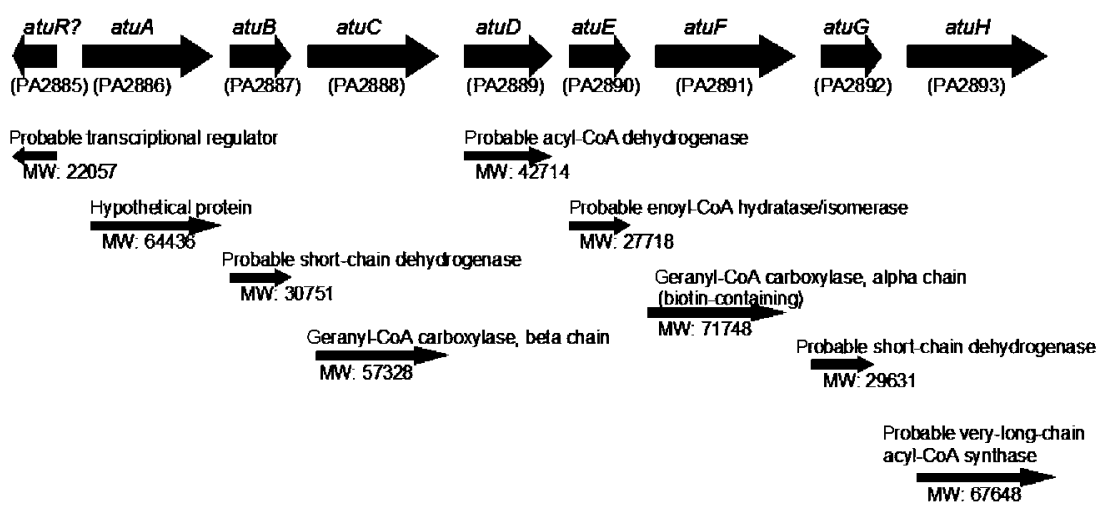

(B) P. aeruginosa liu cluster

(C) P. putida, putative liu cluster

Fig. 3. Gene clusters of $P$. aeruginosa PAO1 (A, B) (atu and liu, respectively), and putative liu cluster of $P$. putida (C) (data and annotations are from http://www.pseudomonas.com and http://www.tigr.org). Note that the involvement of PA2885 $(a t u R$ ?) in Atu is hypothetical, and is not supported by experimental data. The deduced molecular weight (MW) of each gene product in $\mathrm{Da}$ is shown.

respectively, and represent the non-biotin-containing carboxylase subunits (LiuB and AtuC were not separated in the double band no. 4/5). The genes $\operatorname{liuA}$ and $a t u F$ have been annotated as biotin-containing subunits of carboxylases in the P. aeruginosa database (Fig. 3). LiuB and liuD have been proposed to encode GCase (corresponding to gnyA and gnyB; Diaz-Perez et al., 2004), and PA2888 and PA2891 ( $a t u C$ and $a t u F$ ) have been suggested to encode MCase; this is contrary to our findings. Our Western blot results shown in Fig. 2, i.e. the findings that the $71 \mathrm{kDa}$ band was expressed only in citronellate-grown cells, and that the $74 \mathrm{kDa}$ band was expressed in both citronellate- and isovalerate-grown cells, suggest that $\operatorname{liuB}$ and $l i u D$ encode MCase $(74 \mathrm{kDa})$, and that $a t u C$ and $a t u F$ encode GCase (71 kDa). The DNA-deduced molecular masses of LiuD and AtuF are 71283 and $71748 \mathrm{Da}$, respectively. The reason for the discrepancy in the theoretical values compared with the observed apparent molecular masses is unknown, but it is often observed in SDS-PAGE, and may depend on the individual amino acid sequence/composition. 
What could be the reason for the contrary conclusions concerning the assignment of LiuB/LiuD and AtuC/AtuF to MCase and GCase subunits? Diaz-Perez et al. (2004) also noticed the relatedness of LiuB/LiuD and AtuC/AtuF; they assumed that the compound 6-methyl-5-hepten-2-one would enter the combined citronellol/leucine degrading pathway at the level of methylcrotonyl-CoA, and considered growth of insertion mutants in the liu gene cluster on 6methyl-5-hepten-2-one as evidence for the functionality of the leucine utilization pathway. However, it is unclear how catabolism of the eight-carbon-atom-containing compound 6-methyl-5-hepten-2-one concurs with the five-carbonatom compound methylcrotonylate (CoA-derivative). Nevertheless, we tested growth of $P$. aeruginosa PAO1 on 6-methyl-5-hepten-2-one, and, surprisingly, we did not observe significant growth on this compound. We tested six other strains of $P$. aeruginosa (HABS1, CF-3MR, PAC1, B104, PA81 and HAB911), and we used different concentrations and different methods of carbon source application (filter paper soaked in 6-methyl-5-hepten-2-one, and inserted into the lid of the Petri dish; $20 \mu \mathrm{l} 6$-methyl-5hepten-2-one spotted into the space between the bottom of the Petri dish and the agar; and 6-methyl-5-hepten-2-one applied by saturated atmosphere in a sealed container). We did not find growth for any of the seven strains that was stronger than that of controls without the carbon source ( 30 and $37^{\circ} \mathrm{C}$, and periods of up to 1 week, were tested). We concluded that $P$. aeruginosa is unable to use 6-methyl-5hepten-2-one as a sole source of carbon and energy, and that growth on 6-methyl-5-hepten-2-one is not a suitable tool to test the functionality of the Liu pathway in P. aeruginosa. The assay for growth on isovalerate and/or leucine appears to be a more reliable tool for this purpose, since leucine and isovalerate are precursors of methylcrotonyl-CoA in catabolic pathways of many organisms (Fig. 1). Inspection of the genome of another related species, Pseudomonas putida, a bacterium that is able to grow on isovalerate, but unable to utilize citronellol or citronellate, and therefore is unlikely to have the genes for the Atu pathway, revealed the presence of a cluster containing four genes (PP4064-PP4067, Fig. 3). The deduced amino acid sequences of ORFs PP4064PP4067 showed a much higher degree of similarity (67-87\% amino acid identity) to LiuA-LiuE of P. aeruginosa, but only a medium level of similarity to AtuA-AtuH (35-51\% identity). We assume that ORFs PP4064-PP4067 code for several genes of the leucine/isovalerate-degrading pathway in P. putida, and we suggest naming them liu genes (Fig. 3).

To find further experimental evidence for our interpretation, insertion mutagenesis of $a t u F$ (putative GCase biotin subunit, $71 \mathrm{kDa}$ ) was performed as described in Methods. The success of insertion mutagenesis was confirmed by PCR and Southern blot analysis of genomic DNA isolated from the respective mutants (data not shown). When the $P$. aeruginosa mutant with an insertion in atuF was tested for utilization of methyl-branched compounds, no growth was found on aliphatic monoterpenes such as citronellol, citronellate, geraniol and geranylate, confirming the involvement of $a t u F$ in utilization of citronellol and related monoterpenes. However, utilization of isovalerate and leucine was the same in the $a t u F$ mutant as in the wildtype. Growth on substrates unrelated to methyl-branched compounds (e.g. glucose, succinate or acetate) was also not affected. This result clearly shows that MCase and a functional Liu pathway are present in the $a t u F$ insertion mutant (Fig. 1). Accordingly, Western blot analysis of isovalerategrown mutant cells for the presence of biotin-containing proteins showed the expected $74 \mathrm{kDa}$ band, and confirmed that MCase was expressed (Fig. 4). After growth on isovalerate, the biotin-containing proteins of the $a t u F$ mutant were purified by avidin-affinity chromatography. The identity of the isolated $74 \mathrm{kDa}$ band with the liuA gene product, and with the $74 \mathrm{kDa}$ protein of the wild-type, was confirmed by trypsin fingerprint analysis of the avidin-isolated protein. The $63 \mathrm{kDa}$ protein was again identified as PA5435 product (putative acetyl-CoA carboxylase/transcarboxylase subunit). The $71 \mathrm{kDa}$ biotin protein was not found in mutant cells that had been incubated in the presence of citronellate for different time periods (Fig. 4), and this confirmed the absence of GCase in the atuF insertion mutant. The nonbiotin-containing subunits of the purified proteins (58 and $48 \mathrm{kDa}$ ) were identified to correspond to LiuB and PA5436 product by trypsin fingerprint analysis MS (data not shown).

\section{DISCUSSION}

In this study, we continued our investigation of the catabolism of acyclic (mono-) terpenes in P. aeruginosa. A

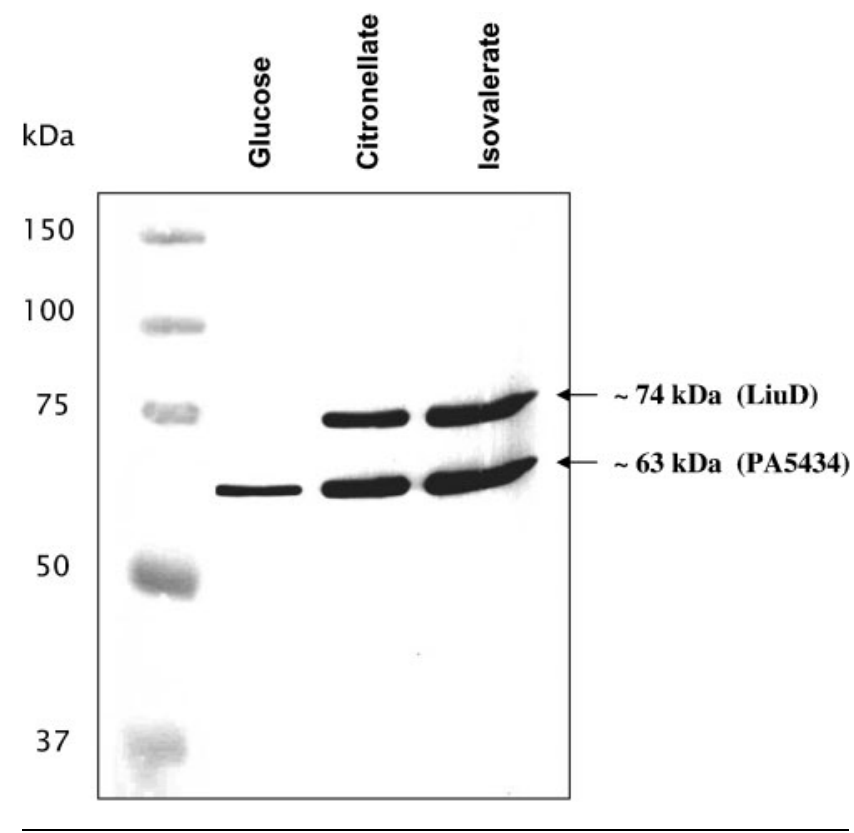

Fig. 4. Western blot analysis of biotin-containing proteins in avidin-purified crude extracts of $P$. aeruginosa (atuF-insertion mutant) grown on glucose $(0.5 \%)$, glucose $(0.1 \%)$ plus citronellate $(0.2 \%)$, and glucose $(0 \cdot 1 \%)$ plus isovalerate $(0.2 \%)$. 
putative pathway leading from citronellol to acetate, acetylCoA and acetoacetate as end products, based on biochemical investigations in P. citronellolis by Seubert and coworkers more than 40 years ago (Seubert, 1960; Seubert et al., 1963; Seubert \& Remberger, 1963; Seubert \& Fass, 1964a, b), is shown in Fig. 1. The pathway includes three phases. (i) Citronellol is first oxidized and activated to geranyl-CoA. Geranyl-CoA is subsequently carboxylated by GCase, hydrated, and the resulting product is cleaved into acetate and 7-methyl-3-oxo-6-octenoyl-CoA (Atu pathway). (ii) 7-Methyl-3-oxo-6-octenoyl-CoA can be degraded by two rounds of subsequent $\beta$-oxidation reactions without hindrance by $\beta$-methyl groups. (iii) The product 3-methylcrotonylCoA concurs with the Liu pathway, which includes a second carboxylation step by MCase. The latter carboxylase is specific for 3-methylcrotonyl-CoA, and is unable to utilize geranyl-CoA as a substrate (Fall \& Hector, 1977). Acetate, acetyl-CoA and acetoacetate are the end products of the combined pathways. While the Atu pathway is present presumably in citronellol-degrading bacteria only ( $P$. citronellolis, $P$. aeruginosa and $P$. mendocina), the Liu pathway probably is present in many organisms, at least in isovalerate- and leucine-utilizing bacteria. The finding that GCase $(71 \mathrm{kDa})$ is expressed only in citronellate-grown cells, and that MCase $(74 \mathrm{kDa})$ is expressed in both citronellategrown and isovalerate-grown cells, is in agreement with the postulated pathway: MCase as a key enzyme of the Liu pathway is needed in equal measure for catabolism of citronellol, and for catabolism of leucine and isovalerate; GCase is needed only for utilization of acyclic (mono-) terpenes such as citronellol and geraniol. MCase can not replace GCase, since it is specific for methylcrotonyl-CoA, and does not accept geranyl-CoA as a substrate (Fall \& Hector, 1977; Hector \& Fall, 1976a, b).

To determine whether the key enzymes GCase and MCase of the combined pathways are expressed in a similar manner in $P$. aeruginosa and $P$. citronellolis, we performed Western blot analysis for biotin-containing proteins (Fig. 2). Indeed, two biotin-containing polypeptides $(74$ and $71 \mathrm{kDa})$ are additionally expressed in citronellate-grown cells compared with glucose-grown cells. Isovalerate-grown cells, however, contain only one additional biotin-containing band $(74 \mathrm{kDa})$ compared with glucose-grown cells. These results show that expression of GCase and MCase in P. aeruginosa is the same as in P. citronellolis. Analysis of the DNA sequences of the biotin-containing subunits of the two candidate carboxylase genes (liuD and $a t u F$ ) shows that the deduced amino acid sequences are highly similar (51\% identity), and that the polypeptides have almost the same theoretical molecular masses of 71.3 and $71.7 \mathrm{kDa}$, respectively. A difference of less than $1 \mathrm{kDa}$ is not sufficient to distinguish the products simply by their migration distance in SDS-PAGE. We therefore identified the respective gene products by trypsin fingerprint analysis, and subsequent ESI-MS ${ }^{n}$ sequencing of isolated peptides. This procedure enabled us to assign the bands in biotin blots to the two citronellate-specific biotin-containing proteins AtuF and LiuD (71 and $74 \mathrm{kDa}$ apparent molecular masses, respectively). This assignment is different from the earlier findings of Diaz-Perez et al. (2004).

Considering the results of Diaz-Perez and coworkers (DiazPerez et al., 2004), and the findings of this contribution, it is evident that both clusters (atuABCDEFGH and liuRABCDE) are essential for catabolism of methyl-branched compounds such as citronellol, citronellate, leucine and isovalerate. We propose to name the cluster PA2886-PA2893 as the atu cluster (acyclic (mono-) terpene utilization, atuABCDEFGH), and the PA2011-PA2016 cluster as the liu gene cluster (leucine and isovalerate utilization, liuRABCDE) (Fig. 3). The name gny (geranoyl), as suggested by Diaz-Perez et al. (2004), does not appear to be suitable for the liu cluster, since our results clearly show that the liu genes are only indirectly involved in the degradation pathway of acyclic terpenes.

Utilization of geraniol apparently requires the presence of additional gene products such as MoeA2, since oxidation of geranial and/or geraniol, in contrast to oxidation of citronellol, includes a molybdenum-dependent step (Höschle \& Jendrossek, 2005). We predict that mutants defective in $l i u D$, and probably most mutants defective in one of the other liu genes, will be shown to be unable to utilize leucine or isovalerate as a carbon source, in addition to the already known minus phenotype on acyclic monoterpenes (DiazPerez et al., 2004).

\section{ACKNOWLEDGEMENTS}

We thank A. Jakob for performing trypsin fingerprint and LC-MS analyses.

\section{REFERENCES}

Burke, Y. D., Stark, M. J., Roach, S. L., Sen, S. E. \& Crowell, P. L. (1997). Inhibition of pancreatic cancer growth by the dietary isoprenoids farnesol and geraniol. Lipids 32, 151-156.

Burke, Y. D., Ayoubi, A. S., Werner, S. R., McFarland, B. C., Heilman, D. K., Ruggeri, B. A. \& Crowell, P. L. (2002). Effects of the isoprenoids perillyl alcohol and farnesol on apoptosis biomarkers in pancreatic cancer chemoprevention. Anticancer Res 22, 3127-3134.

Cantwell, S. G., Lau, E. P., Watt, D. S. \& Fall, R. R. (1978). Biodegradation of acyclic isoprenoids by Pseudomonas species. J Bacteriol 135, 324-333.

Carnesecchi, S., Bradaia, A., Fischer, B., Coelho, D., SchollerGuinard, M., Gosse, F. \& Raul, F. (2002a). Perturbation by geraniol of cell membrane permeability and signal transduction pathways in human colon cancer cells. J Pharmacol Exp Ther 303, 711-715.

Carnesecchi, S., Langley, K., Exinger, F., Gosse, F. \& Raul, F. (2002b). Geraniol, a component of plant essential oils, sensitizes human colon cancer cells to 5-fluorouracil treatment. IARC Sci Publ 156, 407-409.

Carnesecchi, S., Bras-Goncalves, R., Bradaia, A., Zeisel, M., Gosse, F., Poupon, M. F. \& Raul, F. (2004). Geraniol, a component of plant essential oils, modulates DNA synthesis and potentiates 5-fluorouracil efficacy on human colon tumor xenografts. Cancer Lett 215, 53-59.

Diaz-Perez, A. L., Zavala-Hernandez, A. N., Cervantes, C. \& Campos-Garcia, J. (2004). The gnyRDBHAL cluster is involved in 
acyclic isoprenoid degradation in Pseudomonas aeruginosa. Appl Environ Microbiol 70, 5102-5110.

Duncan, R. E., Lau, D., El-Sohemy, A. \& Archer, M. C. (2004). Geraniol and beta-ionone inhibit proliferation, cell cycle progression, and cyclin-dependent kinase 2 activity in MCF-7 breast cancer cells independent of effects on HMG-CoA reductase activity. Biochem Pharmacol 68, 1739-1747.

Fall, R. R. (1981). 3-Methylcrotonyl-CoA and geranyl-CoA carboxylases from Pseudomonas citronellolis. Methods Enzymol 71, 791-799.

Fall, R. R. \& Hector, M. L. (1977). Acyl-coenzyme A carboxylases. Homologous 3-methylcrotonyl-CoA and geranyl-CoA carboxylases from Pseudomonas citronellolis. Biochemistry 16, 4000-4005.

Fall, R. R., Brown, J. L. \& Schaeffer, T. L. (1979). Enzyme recruitment allows the biodegradation of recalcitrant branched hydrocarbons by Pseudomonas citronellolis. Appl Environ Microbiol 38, 715-722.

Hector, M. L. \& Fall, R. R. (1976a). Multiple acyl-coenzyme A carboxylases in Pseudomonas citronellolis. Biochemistry 15, 3465-3472.

Hector, M. L. \& Fall, R. R. (1976b). Evidence for distinct 3methylcrotonyl-CoA and geranyl-CoA carboxylases in Pseudomonas citronellolis. Biochem Biophys Res Commun 71, 746-753.

Hierro, I., Valero, A., Perez, P., Gonzalez, P., Cabo, M. M., Montilla, M. P. \& Navarro, M. C. (2004). Action of different monoterpenic compounds against Anisakis simplex s.l. L3 larvae. Phytomedicine 11, $77-82$.

Höschle, B. \& Jendrossek, D. (2005). Utilization of geraniol is dependent on molybdenum in Pseudomonas aeruginosa: evidence for different metabolic routes for oxidation of geraniol and citronellol. Microbiology 151, 2277-2283.

Izumi, S., Takashima, O. \& Hirata, T. (1999). Geraniol is a potent inducer of apoptosis-like cell death in the cultured shoot primordia of Matricaria chamomilla. Biochem Biophys Res Commun 259, 519-522.
Pawar, P. V., Sharma, R. N., Phadnis, A. P., Nanda, B. \& Patwardhan, S. A. (1991). Action of some insect growth regulators on mosquito vectors: part I - citronellol based diethers. J Commun Dis 23, 118-122.

Rice, P. J. \& Coats, J. R. (1994). Insecticidal properties of several monoterpenoids to the house fly (Diptera: Muscidae), red flour beetle (Coleoptera: Tenebrionidae), and southern corn rootworm (Coleoptera: Chrysomelidae). J Econ Entomol 87, 1172-1179.

Schlegel, H. G., Kaltwasser, H. \& Gottschalk, G. (1961). A submersion method for culture of hydrogen-oxidizing bacteria: growth physiological studies. Arch Mikrobiol 38, 209-222.

Seubert, W. (1960). Degradation of isoprenoid compounds by microorganisms. I. Isolation and characterization of an isoprenoiddegrading bacterium, Pseudomonas citronellolis n. sp. J Bacteriol 79, 426-434.

Seubert, W. \& Fass, E. (1964a). Studies on the bacterial degradation of isoprenoids. V. The mechanism of isoprenoid degradation. Biochem Z 341, 35-44.

Seubert, W. \& Fass, E. (1964b). Studies on the bacterial degradation of isoprenoids. IV. The purification and properties of betaisohexenylglutaconyl-CoA-hydratase and beta-hydroxy-betaisohexenylglutaryl-CoA-lyase. Biochem $Z$ 341, 23-34.

Seubert, W. \& Remberger, U. (1963). Studies on the bacterial degradation of isoprenoid compounds. II. The role of carbon dioxide. Biochem Z 338, 245-264.

Seubert, W., Fass, E. \& Remberger, U. (1963). Studies on the bacterial degradation of isoprenoid compounds. III. Purification and properties of geranyl carboxylase. Biochem $Z$ 338, 265-275.

Simon, R., Priefer, U. \& Pühler, A. (1983). A broad host range mobilization system for in vivo genetic engineering: transposon mutagenesis in Gram-negative bacteria. Bio/Technology 1, 784-791.

Windgassen, M., Urban, A. \& Jaeger, K. E. (2000). Rapid gene inactivation in Pseudomonas aeruginosa. FEMS Microbiol Lett 193, 201-205. 